\title{
Atrial Myxoma Mimicking Mitral Stenosis
}

\author{
Michael Spartalis ${ }^{\mathrm{a}, \mathrm{e}}$, Eleni Tzatzaki ${ }^{\mathrm{a}}$, Eleftherios Spartalis ${ }^{\mathrm{b}}$, Demetrios Moris ${ }^{\mathrm{c}}$, \\ Antonios Athanasiou $^{\text {d, Stamatios Kyrzopoulos }}{ }^{\text {a }}$, Dimitrios Tsiapras ${ }^{\text {a }}$, \\ Panagiotis Kalogris $^{\mathrm{a}}$, Vassilis Voudris ${ }^{\mathrm{a}}$
}

\begin{abstract}
Myxoma is the most common primary cardiac tumor and is usually located in the left atrium. Clinical manifestation relies in large part on the size, location, and architecture of the tumor. There are many reports in the literature of cardiac myxomas causing syncope, embolism, even myocardial infarction. We present a rare case of a patient who underwent urgent surgical resection of a large left atrial myxoma mimicking mitral stenosis. The postoperative course of the patient was uncomplicated. One year after surgery, the patient is asymptomatic and disease-free.
\end{abstract}

Keywords: Cardiac tumors; Myxoma; Mitral stenosis

\section{Introduction}

Myxoma is the most common primary cardiac tumor and is usually located in the left atrium. The tumor is located in the left atrium in $75 \%$ of the cases, in the right atrium in $23 \%$ and $2 \%$ of the cases is found in ventricles. In rare occasions, myxoma is found in more than one chamber. It is more common in women than men, and it can be manifested at any age [1].

Clinical manifestation relies in large part on the size, location, and architecture of the tumor. Myxomas can cause a pulmonary or systematic embolism, depending on the position of the tumor in the heart [2]. It can cause obstruction in the blood flow, mimicking mitral stenosis like in our case or mitral regurgitation as a result of complete blockage of the valve [3, 4]. Non-specific symptoms like fever, weight loss, and fatigue have also been reported [2].

The only treatment is surgical resection with high success

Manuscript submitted May 22, 2017, accepted May 26, 2017

aDivision of Cardiology, Onassis Cardiac Surgery Center, Athens, Greece bLaboratory of Experimental Surgery and Surgical Research, University of Athens, Medical School, Athens, Greece

'Department of Surgery, The Ohio State University Comprehensive Cancer Center, The Ohio State University, Columbus, OH, USA

${ }^{\mathrm{d} D e p a r t m e n t}$ of Surgery, Mercy University Hospital, Cork, Ireland

${ }^{\mathrm{e} C}$ Corresponding Author: Michael Spartalis, Division of Cardiology, Onassis

Cardiac Surgery Center, Fokidos 42, Athens 115 27, Greece.

Email:msparta@med.uoa.gr

doi: https://doi.org/10.14740/cr558w rates, and the recurrences are rare $[2,5]$.

\section{Case Report}

A 43-year-old patient with complaints of multiple syncopal episodes and severe exertion presented to our emergency department with acute pulmonary edema. Echocardiography revealed severe dilatation of the left atrium and an enormous mass attached to the interatrial septum reaching the atrial surface of the mitral valve (Figs. 1 and 2).

The mass occupied the entire left atrium protruding into the left ventricle during the opening of the mitral valve causing severe mitral stenosis with max PG $12 \mathrm{~mm} \mathrm{Hg}$ (Fig. 3).

The patient underwent urgent surgical resection. A median sternotomy was performed, followed by right atriotomy. Inside the left atrium, an enormous mass was observed, overlapped by a thrombus ( $7 \mathrm{~cm}$ total diameter). The mass was resected without complications, and the septal defect was closed with a pericardial patch.

Histology showed myxoid matrix with stellate or elongated polygonal cells, scattered lymphocytes and red blood cells in keeping with myxoma (Fig. 4).

\section{Discussion}

Myxomas are easily misdiagnosed and should be considered

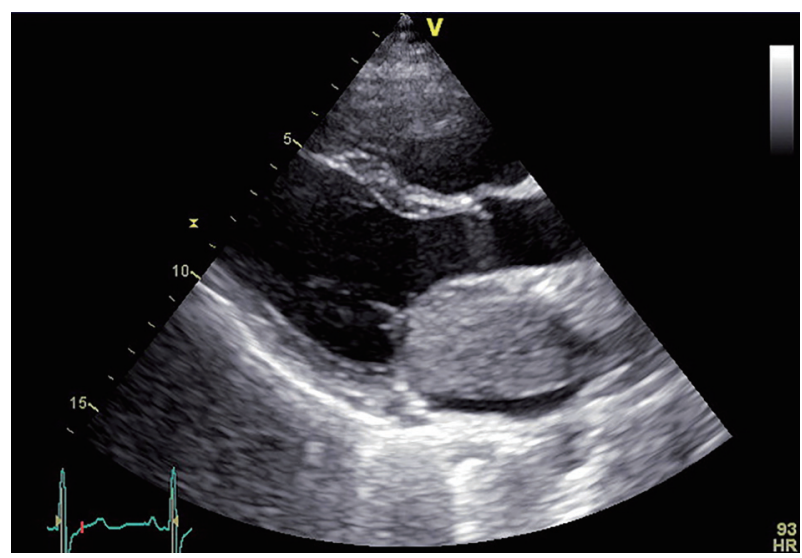

Figure 1. Long parasternal axis view of transthoracic echocardiography showing a left atrial myxoma reaching the atrial surface of the mitral valve. 


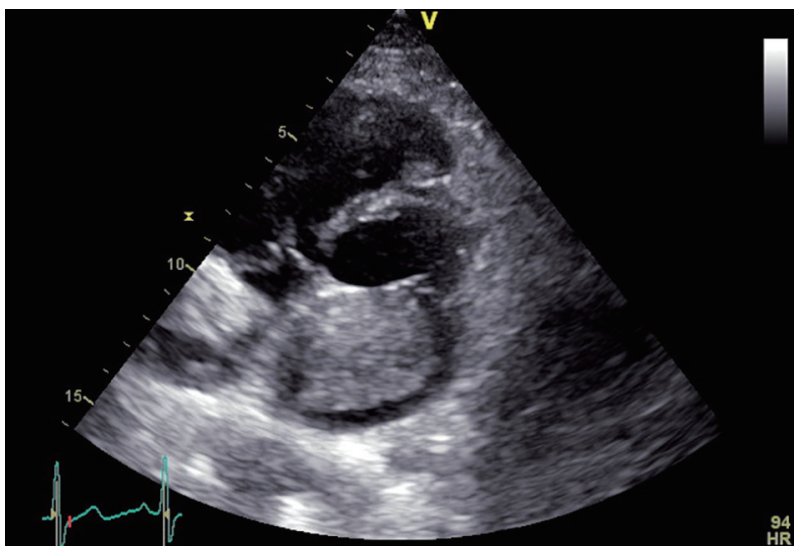

Figure 2. Short parasternal axis view of transthoracic echocardiography demonstrating a left atrial myxoma covering the entire left atrium.

in the differential diagnosis in patients with suspected mitral valve disease $[1,3]$. Echocardiography should be performed as early as possible to establish a prompt diagnosis and management [6].

Due to a variety of non-specific findings at first presentation, myxoma is initially suspected in only $5.7 \%$ of patients. Clinical presentation consists of cardiac (67\%), embolic (29\%) and systemic (34\%) symptoms, even sudden cardiac death. Symptoms of heart failure, as in the current case, are typically caused by partial obstruction of the mitral valve orifice by the myxoma [2].

Sudden cardiac death in patients with a primary cardiac tumor is rare and is estimated around $0.01-0.005 \%$ of all sudden deaths. Association between sudden cardiac death and cardiac myxoma has been reported in as early as 1953 by Madonia et al. A review of the literature on this subject between 1950 and 2017 revealed 17 cases of sudden death attributed to cardiac myxoma in adults [4].

Sudden death in myxoma is attributed to either severe acute disturbance in cardiac hemodynamics from cardiac obstruction to coronary embolization from the tumor [7]. The latter is probably responsible for sudden death in patients

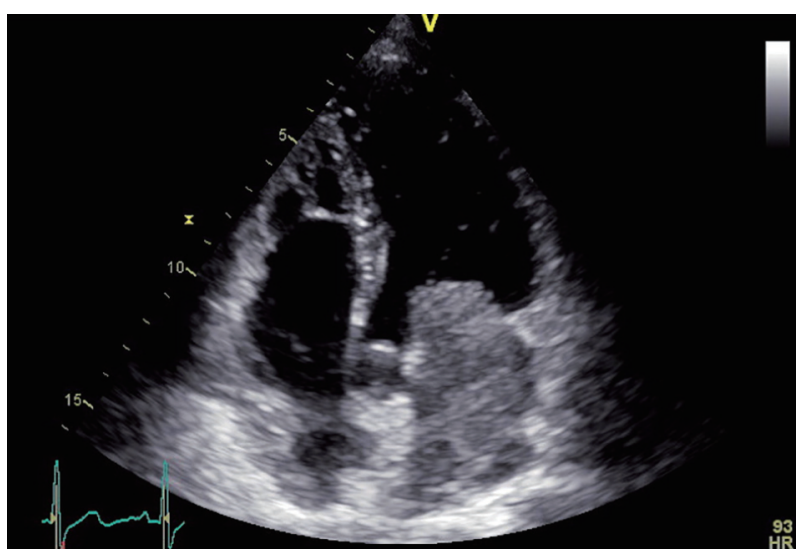

Figure 3. Apical four-chamber view of transthoracic echocardiography showing a left atrial myxoma attached to the interatrial septum and dilated ventricles.

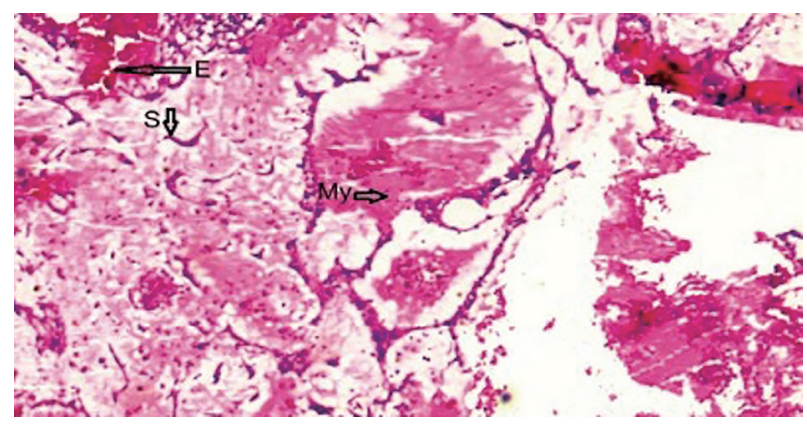

Figure 4. Hematoxylin and eosin staining method (magnification ratio, $\times 20$ ) showing myxoid matrix with stellate or elongated polygonal cell (My), scattered lymphocytes (S) and red blood cells (E).

with very small tumors. All of the above suggest that early surgery is essential and once it has been identified that a patient has a myxoma, that is significant enough to cause complete intracardiac obstruction, it must be performed urgently [5].

\section{Conclusions}

Surgical removal is the method of choice for the treatment of cardiac tumors such as myxoma. Excision helps to reduce complications, as myxomas increase rapidly in size and cause obstructive and embolic phenomena.

\section{Consent}

Written informed consent was obtained from the patient for publication of this case report and accompanying images.

\section{Financial Support}

This research received no specific grant from any funding agency, commercial or not-for-profit sectors.

\section{Conflicts of Interest}

All authors have contributed and approved the final version of this manuscript. No author has any conflict of interest to disclose.

\section{References}

1. El Sabbagh A, Al-Hijji MA, Thaden JJ, Pislaru SV, Pislaru C, Pellikka PA, Arruda-Olson AM, et al. Cardiac Myxoma: The Great Mimicker. JACC Cardiovasc Imaging. 2017;10(2):203-206.

2. Pinede L, Duhaut P, Loire R. Clinical presentation of left atrial cardiac myxoma. A series of 112 consecutive cases. Medicine (Baltimore). 2001;80(3):159-172. 
3. Anandan PK, Bengaluru Hanumanthappa N, Bhat P, Manjunath CN, Chandrasekaran D. Ping-Pong Mitral Stenosis: Left Atrial Myxoma With Mitral Stenosis and Pulmonary Hypertension in an Octogenarian. Cardiol Res. 2015;6(3):286-288.

4. Madonia PF, Boggiano R, Gubner R. Ball-valve syndrome caused by primary cardiac tumor. N Y State J Med. 1953;53(24):3043-3044.

5. Ren DY, Fuller ND, Gilbert SA, Zhang Y. Cardiac Tu- mors: Clinical Perspective and Therapeutic Considerations. Curr Drug Targets. 2016.

6. Oliveira R, Branco L, Galrinho A, Abreu A, Abreu J, Fiarresga A, Mamede A, et al. Cardiac myxoma: a 13-year experience in echocardiographic diagnosis. Rev Port Cardiol. 2010;29(7-8):1087-1100.

7. Panidis IP, Mintz GS, McAllister M. Hemodynamic consequences of left atrial myxomas as assessed by Doppler ultrasound. Am Heart J. 1986;111(5):927-931. 